\title{
Multiple Sclerosis Presenting with Sixth Nerve Palsy in a Child
}

\author{
Nada Al-Yousuf \\ Lolwah Aljutaili ${ }^{2}$ \\ Aisha AlHuwais ${ }^{2}$ \\ Abdullah Almutairi ${ }^{2}$ \\ Hasan Alsetri ${ }^{3}$ \\ Moiz Bakhiet $\mathbb{D}^{4}$ \\ 'Department of Ophthalmology, King \\ Abdullah Medical City, Manama, Kingdom \\ of Bahrain; ${ }^{2}$ College of Medicine \& \\ Medical Sciences, Arabian Gulf University, \\ Manama, Kingdom of Bahrain; \\ ${ }^{3}$ Department of Chemistry \& \\ Biochemistry, University of California Los \\ Angeles, Los Angeles, CA, USA; \\ ${ }^{4}$ Department of Molecular Medicine, \\ College of Medicine \& Medical Sciences, \\ Arabian Gulf University, Manama, \\ Kingdom of Bahrain
}

Correspondence: Nada Al-Yousuf Department of Ophthalmology, King Abdullah Medical City, 6I, King Abdulaziz Avenue, Manama, Bahrain

Tel $+9737731007 \mid$

Fax +97377310001

Email nyousufl0@gmail.com

\begin{abstract}
We report a child with multiple sclerosis who presented with sixth nerve palsy. She is a twelve-year-old Bahraini female presented to the ophthalmology department complaining of double vision. She also had imbalance and paraesthesia. Extraocular muscle examination showed restriction of abduction in the right eye. There was no change in vision. MRI showed right eye optic neuritis $(\mathrm{ON})$ and demyelination which was indicative of multiple sclerosis (MS). Ocular coherence tomography (OCT) showed thinning of nerve fibres of both eyes which was consistent with subclinical optic neuritis. Neurological examination showed brisk knee jerk on the left side and incoordination of movement on the same side. Disability Status Scale (EDSS) showed a score of 3.0. She was given Solumedrol $500 \mathrm{mg}$ intravenously (IV) in addition to omeprazole $40 \mathrm{mg}$ IV and Vitamin D3 (cholecalciferol) 50,000 IU cap weekly for 8 weeks and Neurorubine forte tablets (vitamin B1, 6, 12) once a day for 2 months. She became asymptomatic in her follow-up visits. Children with MS can present as sixth cranial nerve palsy. Clinicians should have a high index of suspicion for early diagnosis and treatment. In addition to MRI, OCT is a useful diagnostic tool for optic neuritis and MS especially in children.
\end{abstract}

Keywords: demyelination, ocular coherence tomography, lateral rectus palsy, diplopia

\section{Introduction}

Multiple sclerosis (MS) was first described in 1868 by French neurologist JeanMartin Charcot. ${ }^{1}$ MS is considered as the most common immune disorder affecting the central nervous system. ${ }^{2}$ It affects around 2.3 million people globally. ${ }^{3}$ Although it is more commonly seen farther from the equator, some ethnic groups, staying far from the equator, have low prevalence. ${ }^{4-6}$ Indeed, some ethnic groups staying close to the equator were shown to have a relatively high risk of this disease. $^{7,8}$ Data from the Middle East shows that the prevalence of MS is around 51.52/100,000, ranging from 14.77/100,000 in Kuwait to 101.4/100,000 in Turkey. ${ }^{9}$

The incidence of MS in children and in patients below the age of 16 is reported to be $2.7-5 \%$ of the total cases. ${ }^{10}$ Clinical presentations of MS in children include optic neuritis (ON), gait disorders, sensory and motor deficits and brainstem disturbances. $^{11,12}$ Compared to adults, it was reported that children were more likely to have ocular symptoms. ${ }^{13,14}$

Extraocular muscle weakness is not commonly reported in MS. Sixth nerve palsy is a rare presentation of adult MS. ${ }^{14-16}$ Children reported with sixth nerve palsy is mostly due to neoplasms or trauma. ${ }^{17}$

We report herewith a child with MS who presented with sixth nerve palsy, subclinical optic neuritis and neurological symptoms. To the best of our knowledge, 
this is the first case of pediatric multiple sclerosis presenting as sixth nerve palsy to be reported from the Middle East region. Written informed consent was provided by the patient's father to have the case details and photographs published. Approval was required and was obtained from the research committee of Habib Medical Group, the administrative body that is managing the clinical aspect of King Abdullah Medical City, to publish this case report.

\section{Case Report}

The patient is a twelve-year-old Bahraini female presented to the ophthalmology department complaining of double vision since one week. The diplopia was mainly on looking straight and on right gaze. She had no prior viral illness. She was fully vaccinated. She has an error of refraction and uses glasses. Ophthalmic examination showed the following: Visual acuity unaided was 0.7 in the right eye and 0.7 in the left eye. There was no relative afferent pupillary defect (RAPD). Slit lamp ophthalmic examination showed clear corneas, normal anterior chamber, normal lens and iris pattern. Fundus examination showed normal maculae and no swelling of the optic discs of both eyes. Extraocular muscle examination showed restriction of abduction in the right eye. The other eye movements were full (Figure 1). Convergence was poor. Orthoptic examination was performed. Worth's Fourth Dot test showed suppression of the right eye. Stereopsis test using random-dot stereogram was 800 seconds of arc only. Titmus stereotest was also abnormal. Krimsky test showed a deviation angle of 20 prism dioptre of esotropia in the right eye for near and distance vision. Performing subjective refraction, the right eye, with -0.75 cylinder correction at 10 degrees, her vision was 0.8 . Performing subjective refraction, the left eye, with -0.75 spherical correction, her vision was 0.8 . Ocular coherence tomography (OCT) was done. It showed thinning of the retinal nerve fibre layer (RNFL) of both eyes. The average RNFL thickness is asymmetrical in both eyes with $58 \%$ of asymmetry. The RNFL in the nasal quadrant of the right eye and left eye are $68 \mu \mathrm{m}$ and 59 $\mu \mathrm{m}$, respectively. The RNFL in the temporal quadrant of the left eye is $55 \mu \mathrm{m}$ (Figure 2). MRI of brain was done. Pre- and post-intravenous contrast multiplanar and multisequential MRI study of the brain was performed. Multiple periventricular and deep white matter nodular small foci
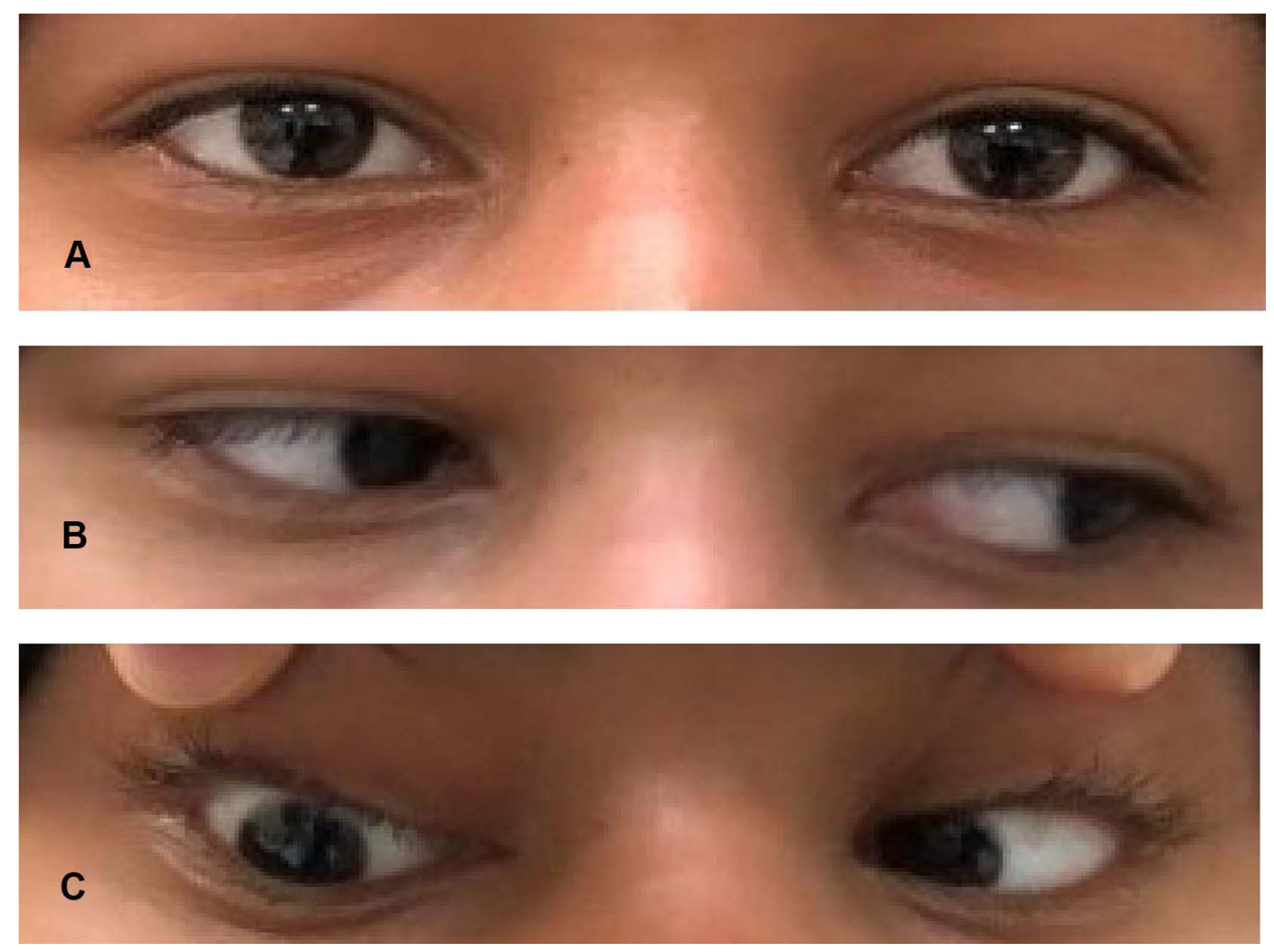

Figure I The patient's extraocular eye movements in different gaze positions. (A) The patient is looking straight showing no limitation of extraocular muscles. (B) The patient is looking to the left. There is no limitation of extraocular muscle movements. (C) The patient is looking to the right. There is limitation of abduction of the right eye indicating weakness of the right lateral rectus muscle due to sixth nerve palsy. 


\section{\begin{tabular}{ll|l}
\hline ONH and RNFL OU Analysis:Optic Disc Cube 200x200 OD O & OS \\
\hline
\end{tabular}}

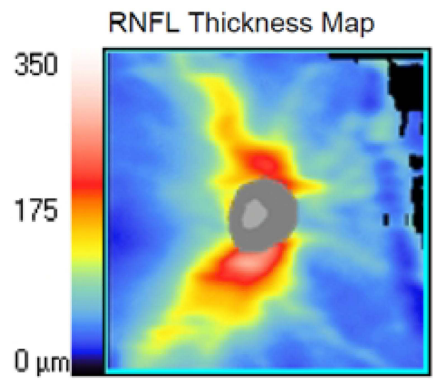

RNFL Deviation Map

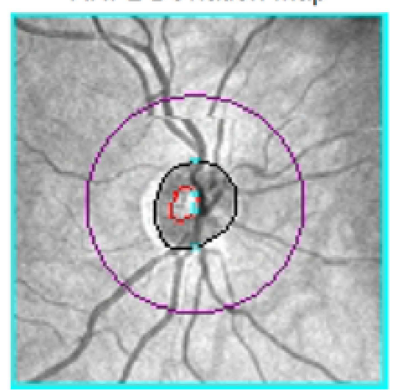

Disc Center $(-0.09,-0.03) \mathrm{mm}$

Extracted Horizontal Tomogram

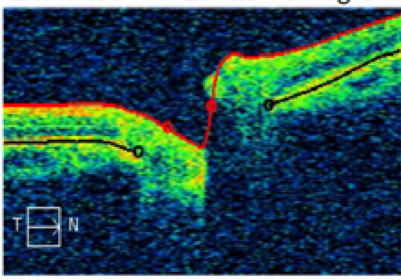

Extracted Vertical Tomogram

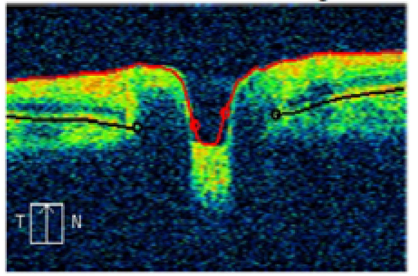

RNFL Circular Tomogram

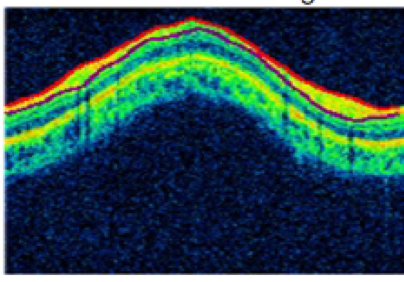

\begin{tabular}{|r|c|c|}
\hline & OD & oS \\
\hline Average RNFL Thickness & $88 \mu \mathrm{m}$ & $82 \mu \mathrm{m}$ \\
\hline RNFL Symmetry & \multicolumn{2}{|c|}{$58 \%$} \\
\hline Rim Area & $1.27 \mathrm{~mm}^{2}$ & $1.04 \mathrm{~mm}^{2}$ \\
\hline Disc Area & $1.47 \mathrm{~mm}^{2}$ & $1.45 \mathrm{~mm}^{2}$ \\
\hline Average CD Ratio & 0.37 & 0.53 \\
\hline Vertical CD Ratio & 0.36 & 0.52 \\
\hline Cup Volume & $0.052 \mathrm{~mm}^{3}$ & $0.122 \mathrm{~mm}^{3}$ \\
\hline
\end{tabular}

Neuro-retinal Rim Thickness

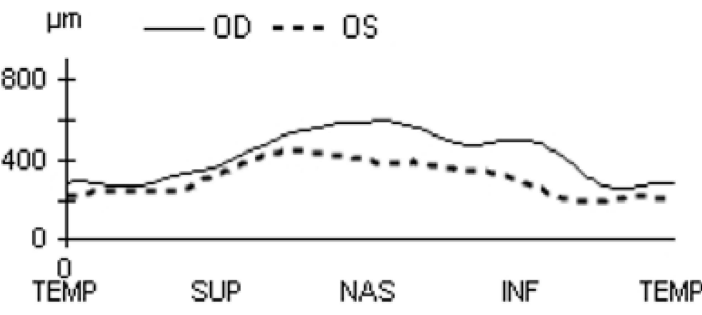

RNFL Thickness

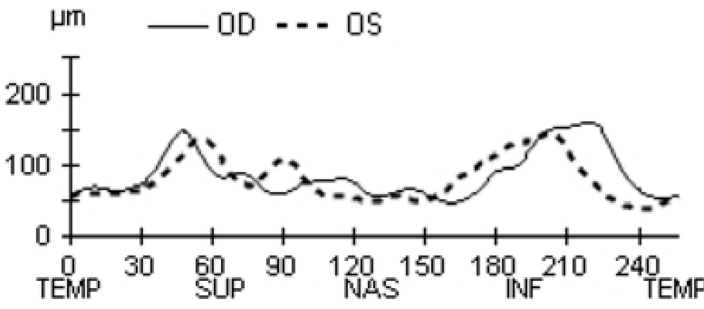

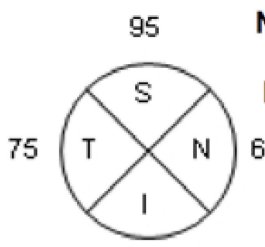

112

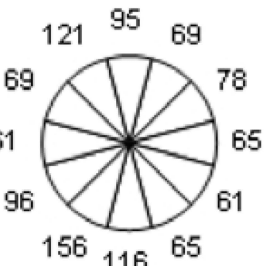

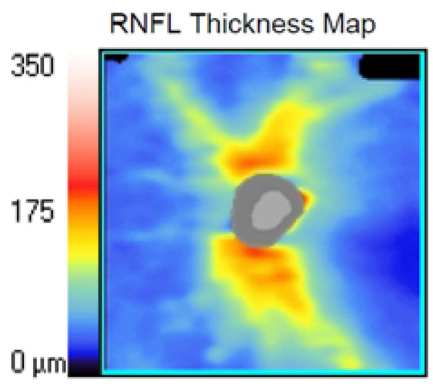

RNFL Deviation Map

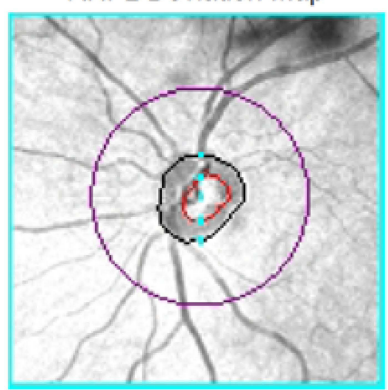

Disc Center $(0.03,0.09) \mathrm{mm}$

Extracted Horizontal Tomogram

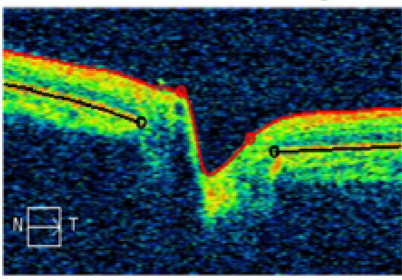

Extracted Vertical Tomogram

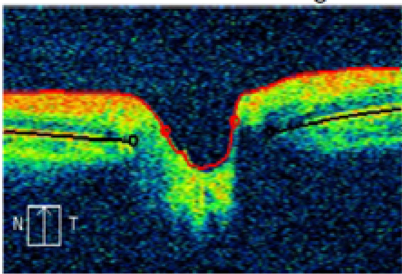

RNFL Circular Tomogram

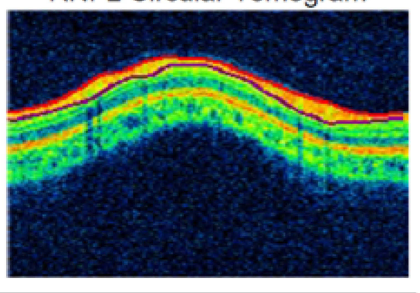

Figure 2 Average RNFL thickness is asymmetrical in both eyes with $58 \%$ of asymmetry. RNFL thickness values for different locations of the optic nerve scans showing the average thickness in $\mu \mathrm{m}$ in superior, temporal, inferior and nasal quadrants. The RNFL in the nasal quadrant of the right eye and left eye are $68 \mu \mathrm{m}$ and $59 \mu \mathrm{m}$, respectively. Both readings are significantly thin compared with normal controls $(96 \mu \mathrm{m})$. The RNFL in the temporal quadrant of the left eye is $55 \mu \mathrm{m}$ which is severely thin compared with normal controls $(73 \mu \mathrm{m})$. This thinning of RNFL quadrants is consistent with ON.

Abbreviations: OD, right eye; OS, left eye; RNFL, retinal nerve fibre layer; ON, optic neuritis; S, superior; T, temporal; I, inferior; N, nasal. 
are seen in both cerebral hemispheres including temporal lobes. Nodular foci were seen at left frontal high periventricular, left peritrigonal and posterosuperior to right Sylvia fissure, left anteromedial margin of midbrain, and left superior cerebellar peduncle. There was no intra- or extraaxial collection of degraded blood products. Size and shape of ventricles, basal cistern and cortical sulci were normal; no midline shift; no posterior fossa masses; no cerebellopontine angle masses.; and normal cranio-cervical junction. The MRI features were suggestive of demyelinating disease and optic neuritis (Figure 3). The impression was multiple sclerosis with sixth nerve palsy and optic neuritis. The patient was referred to neurology.

She had neurological symptoms, in addition to diplopia, she had imbalance and paraesthesia. She had no fever, headache or vomiting. There was no altered level of consciousness, behavioral changes, acute cognitive dysfunction, and no seizures. There were no signs of decreased voluntary movement, muscle weakness or incoordination of movement. Disability was evaluated using the Kurtzke Expanded Disability Status Scale (EDSS), and she had a score of 3.0 with mild disability in three functional systems (FS). Blood test for NMO antibodies was negative. Erythrocyte sedimentation rate (ESR) and C-reactive protein (CRP) were within normal levels.

The diagnosis of MS was made. The patient was given Solu-medrol $500 \mathrm{mg}$ intravenously (IV), in addition to omeprazole $40 \mathrm{mg}$ IV and Vitamin D3 (cholecalciferol) 50,000 IU cap weekly for 8 weeks. Neurorubine forte tablets (vitamin B1, 6, 12) were prescribed once a day for 2 months. The patient received a tapering dose of prednisolone $30 \mathrm{mg}$ for 3 days and then reduced by $5 \mathrm{mg}$ every 3 days with $20 \mathrm{mg}$ of Nexium tablets daily. One week later, the patient was reassessed and her EDSS went down to 1.0 with no disability and minimal signs in one FS. Patient was put on interferon-beta treatment as a disease-modifying drug (DMD) and regularly followed
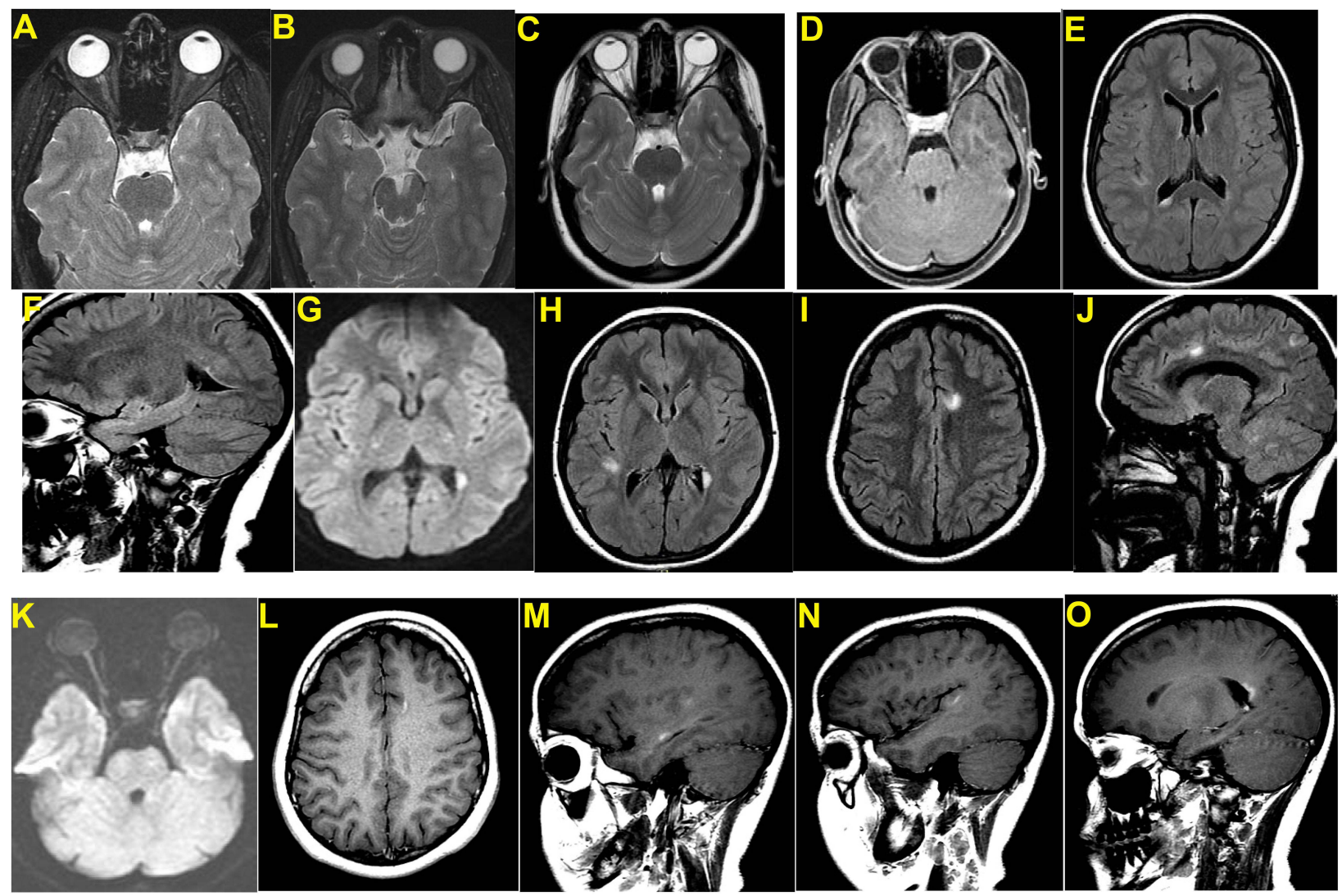

Figure 3 MRI of brain. (A) T2 fat sat image right optic nerve hyperintensity indicating optic neuritis. (B) T2 axial image showing left cerebral peduncle plaque. (C) T2 axial showing right optic neuritis. (D) TI axial post-contrast image showing patchy enhancement of right optic nerve. (E) Periventricular T2 hyperintense focus in right peritrigonal region. (F) Flair sagittal image showing ependymal dot-dash sign sensitive in early MS. (G) Diffusion-weighted image showing left peri ventricular bright focus- T2 shine through effect. (H) Flair axial image showing right parietal juxtacortical and left periventricular foci. (I) Flair axial image showing left parafalcine frontal juxtacortical focus. (J) Sagittal flair image showing juxtacortical and infratentorial plaques. (K) Diffusion-weighted image showing bright right optic nerve. (L-N) Open ring enhancement of juxtacortical plaque. (O) Nodular enhancement in periventricular plaque. 
at the neurological clinic. Six months later, she was fully recovered symptomatically.

\section{Discussion}

OCT is a useful tool in ON and MS which may reveal thinning in the RNFL in the absence of clinical findings. ${ }^{18,19}$

OCT is a non-contact scanning machine that provides cross-sectional visualization of the optic nerve head and measurements of retinal nerve fiber layer (RNFL) thickness in different quadrants of the optic nerve. It has the advantage of being noninvasive and can easily be performed in pediatric age group. Unlike MRI, OCT does not require sedation in children. It is particularly useful in MS, in which the anterior visual pathways are commonly affected. RNFL thickness values for different locations of the optic nerve scans show the average thickness in $\mu \mathrm{m}$ in superior, temporal, inferior and nasal quadrants. The patient's RNFL thicknesses in the nasal quadrant of the right eye and left eye are $68 \mu \mathrm{m}$ and $59 \mu \mathrm{m}$, respectively. Both readings are significantly thin compared with normal controls $(96 \mu \mathrm{m}){ }^{20}$ The RNFL in the temporal quadrant of the left eye is 55 $\mu \mathrm{m}$, which is severely thin compared with normal controls $(73 \mu \mathrm{m}) .^{20}$ This thinning of RNFL quadrants is consistent with $\mathrm{ON}$.

Our patient had no RAPD and no OD swelling, yet her OCT results showed thinning in RNFL in both eyes.

Interestingly, although the MRI findings suggested right eye optic neuritis, there was bilateral thinning of the RNFL on OCT, more in the left eye. This finding sheds light on the importance of performing both MRI and OCT when investigating children with MS. RNFL thinning can be evident in the absence of clinical and MRI findings.

It is not uncommon for children with MS to present with ON. However, sixth nerve palsy, as a presenting sign in pediatric MS, is rare. ${ }^{21-23}$

A series of cases of pediatric MS were reported from Taiwan, none of them presented as sixth nerve palsy. ${ }^{24}$ Isolated sixth nerve palsy in children without MS was reported in the medical literature. ${ }^{25,26}$ Searching the literature for pediatric MS with sixth nerve palsy, one case was reported from Brazil, a child with MS who presented with diplopia and convergent strabismus and found to have sixth nerve palsy. ${ }^{27}$

\section{Conclusion}

Pediatric multiple sclerosis can present as sixth cranial nerve palsy. Clinicians should have a high index of suspicion for early diagnosis and treatment. In addition to MRI, optic neuritis can be diagnosed by OCT, even in the absence of clinical signs.

Future studies to investigate MRI and OCT correlation in optic neuritis, especially in children, are recommended.

\section{Acknowledgment}

We thank Dr Jameel Ahmed, consultant radiologist at King Abdullah Medical City, for providing us with additional MRI sections with detailed explanations.

\section{Disclosure}

The authors report no conflicts of interest in this work.

\section{References}

1. Charcot M. Histologie de la sclérose en plaques. Gaz Hosp. 1868;41:554-556

2. Berer K, Krishnamoorthy G. Microbial view of central nervous system autoimmunity. FEBS Lett. 2014;588(22):4207-4213. doi:10.1016/j.febslet.2014.04.007

3. Disease GBD, Injury I, Prevalence C. Global, regional, and national incidence, prevalence, and years lived with disability for 310 diseases and injuries, 1990-2015: a systematic analysis for the Global Burden of Disease Study 2015. Lancet. 2016;388(10053):1545-1602.

4. Compston A, Coles A. Multiple sclerosis. Lancet. 2008;372 (9648):1502-1517. doi:10.1016/S0140-6736(08)61620-7

5. Alonso A, Hernan MA. Temporal trends in the incidence of multiple sclerosis: a systematic review. Neurology. 2008;71(2):129-135. doi:10.1212/01.wnl.0000316802.35974.34

6. Pugliatti M, Sotgiu S, Rosati G. The worldwide prevalence of multiple sclerosis. Clin Neurol Neurosurg. 2002;104(3):182-191. doi:10.1016/S0303-8467(02)00036-7

7. Milo R, Kahana E. Multiple sclerosis: geoepidemiology, genetics and the environment. Autoimmun Rev. 2010;9(5):A387-394. doi:10.1016/ j.autrev.2009.11.010

8. Grimaldi LM, Salemi G, Grimaldi G, et al. High incidence and increasing prevalence of MS in Enna (Sicily), Southern Italy. Neurology. 2001;57(10):1891-1893. doi:10.1212/WNL.57.10.1891

9. Heydarpour P, Khoshkish S, Abtahi S, et al. Multiple sclerosis epidemiology in Middle East and North Africa: a systematic review and meta-analysis. Neuroepidemiology. 2015;44(4):232-244. doi:10.115 9/000431042

10. Boiko A, Vorobeychik G, Paty D, et al. Early onset multiple sclerosis: a longitudinal study. Neurology. 2002;59(7):1006-1010. doi:10.1212/ WNL.59.7.1006

11. Simone IL, Carrara D, Tortorella C, et al. Course and prognosis in early-onset MS: comparison with adult-onset forms. Neurology. 2002;59(12):1922-1928. doi:10.1212/01.WNL.0000036907.37650.8E

12. Ozakbas S, Idiman E, Baklan B, et al. Childhood and juvenile onset multiple sclerosis: clinical and paraclinical features. Brain Dev. 2003;25(4):233-236. doi:10.1016/S0387-7604(03)00034-2

13. Kaufmann DI. Multiple sclerosis and the eye. Ophthalmol Clin North Am. 1992;5:513-531.

14. Thomke F, Lensch E, Ringel K, et al. Isolated cranial nerve palsies in multiple sclerosis. J Neurol Neurosurg Psychiatry. 1997;63 (5):682-685. doi:10.1136/jnnp.63.5.682

15. Barr D, Kupersmith MJ, Turbin R, et al. Isolated sixth nerve palsy: an uncommon presenting sign of multiple sclerosis. J Neurol. 2000;247 (9):701-704. doi:10.1007/s004150070114 
16. Muhammed K, Ball J. Multiple sclerosis causing a partial sixth nerve palsy. BMJ Case Rep. 2014;2014:bcr2013201239.

17. Park KA, Oh SY, Min JH, et al. Acquired onset of third, fourth, and sixth cranial nerve palsies in children and adolescents. Eye (Lond). 2019;33(6):965-973. doi:10.1038/s41433-019-0353-y

18. Lamirel C, Newman NJ, Biousse V. Optical coherence tomography (OCT) in optic neuritis and multiple sclerosis. Rev Neurol (Paris). 2010;166(12):978-986. doi:10.1016/j.neurol.2010.03.024

19. Graves JS, Chohan H, Cedars B, et al. Sex differences and subclinical retinal injury in pediatric-onset MS. Mult Scler. 2017;23(3):447-455. doi: $10.1177 / 1352458516652497$

20. Yanni SE, Wang J, Cheng CS, et al. Normative reference ranges for the retinal nerve fiber layer, macula, and retinal layer thicknesses in children. Am J Ophthalmol. 2013;155(2):354-360 e351. doi:10.1016/ j.ajo.2012.08.010

21. Sivaraman I, Moodley M. Multiple sclerosis in the very young: a case report and review of the literature. Neurodegener Dis Manag. 2016;6 (1):31-36. doi:10.2217/nmt.15.70
22. Suppiej A, Cainelli E. Cognitive dysfunction in pediatric multiple sclerosis. Neuropsychiatr Dis Treat. 2014;10:1385-1392. doi:10.2147/NDT.S48495

23. Domingo R, Martinez-Salcedo E, Climent V, et al. [Multiple sclerosis: a case report of early onset]. Rev Neurol. 1999;28(5):488-491. Portuguese.

24. Weng WC, Yang CC, Yu TW, et al. Multiple sclerosis with childhood onset: report of 21 cases in Taiwan. Pediatr Neurol. 2006;35 (5):327-334. doi:10.1016/j.pediatrneurol.2006.05.002

25. Gonçalves R, Coelho P, Menezes C, Ribeiro I. Benign recurrent sixth nerve palsy in a child. Case Rep Ophthalmol Med. 2017;2017:8276256. doi:10.1155/2017/8276256

26. Mahoney NR, Liu GT. Benign recurrent sixth (abducens) nerve palsies in children. Arch Dis Child. 2009;94(5):394-396. Epub 2009 Jan 8. PMID: 19131423. doi:10.1136/adc.2008.142794

27. de Albuquerque RC, de Paula RS, Brito MM, et al. Pediatric multiple sclerosis - a challenging demyelinating disease: case report and brief review of the literature. Case Rep Pediatr. 2012;2012:684064.

\section{Publish your work in this journal}

The International Medical Case Reports Journal is an international, peer-reviewed open-access journal publishing original case reports from all medical specialties. Previously unpublished medical posters are also accepted relating to any area of clinical or preclinical science. Submissions should not normally exceed 2,000 words or 4 published pages including figures, diagrams and references. The manuscript management system is completely online and includes a very quick and fair peer-review system, which is all easy to use. Visit http://www.dovepress.com/testimonials.php to read real quotes from published authors. 